\title{
Management of COVID-19-Positive Patient with Bilateral Longitudinal Petrous Fractures: A Case Report
}

\author{
Mohamed Ragab Nagy ${ }^{1}$ Mohamed Abdelrahman Ibrahim²
}

\author{
${ }^{1}$ Department of Neurosurgery, Faculty of Medicine, Cairo \\ University, Kasr Al Ainy Street, Cairo Governorate, Egypt \\ ${ }^{2}$ Department of Infection Prevention and Control, Najran Armed \\ Forces Hospital, Najran, Kingdom of Saudi Arabia
}

\begin{abstract}
Address for correspondence Mohamed Ragab Nagy, MD, Department of Neurosurgery, Faculty of Medicine, Cairo University, Cairo, Egypt (e-mail: dr_mohamedragab@hotmail.com).
\end{abstract}

Indian J Neurotrauma:2021;18:75-78

\begin{abstract}
Temporal bone fractures are bilateral in 8 to $29 \%$ of cases. High index of suspicion of coronavirus disease 2019 (COVID-19) infection is important while dealing with Keywords

- bilateral longitudinal petrous fractures

- management

- COVID-19

- sensorineural hearing loss

- cerebrospinal

fluid leak any patient with special concern to the surgical and traumatized patients to reach a good patient outcome especially during the pandemic of COVID-19. We describe in this report the management of COVID-19-positive patient with bilateral longitudinal petrous fractures and moderate head injury.
\end{abstract}

\section{Introduction}

Temporal bone fractures are bilateral in 8 to $29 \%$ of cases. They are mostly encountered after road traffic accidents (RTA) followed by assaults and falls. ${ }^{1}$ They are classified into longitudinal, transverse, and oblique (mixed) fractures relative to the plane of the petrous ridge. The most common complications encountered after these fractures are facial nerve injury, cerebrospinal fluid (CSF) leak, and hearing loss. A head computed tomography (CT) usually provides sufficient details for the diagnosis of most temporal bone fractures. ${ }^{2}$

The World Health Organization (WHO) announced that the coronavirus disease 2019 (COVID-19) infection represented a global pandemic on March 12, 2020. ${ }^{3}$ Severe acute respiratory syndrome coronavirus 2 (SARS-CoV-2), which causes COVID-19, is a highly contagious virus with devastating impacts for the healthcare systems everywhere. Since then, healthcare workers have been implementing strategies to try to contain the infection while protecting their patients' and their own health as well.
We described in this case report the management of COVID-19-positive patient with bilateral longitudinal petrous fracture and moderate head injury. To the best of our knowledge, this is the first report about bilateral temporal fractures in COVID-19-positive patients.

\section{Case Report}

A 31-year-old-male patient presented to the emergency department after being involved in RTA by disturbed conscious level, bleeding from the left ear. On presentation, his Glasgow Coma Scale (GCS) was 10/15 (M5, V3, E2), pupils were equal reactive bilaterally, and the motor power of all limbs was intact. ENT examination revealed bleeding from the left ear (CSF leak), for which sterile dressing of the ear was done, and right hemotympanum. The facial nerve could not be assessed at that time.

On presentation, initial CT scan revealed right-sided cerebral edema, right temporoparietal subarachnoid hemorrhage, right temporoparietal small brain contusions, bilateral petrous bones longitudinal fractures, nondisplaced fracture published online

October 9, 2020
DOI https://doi.org/

$10.1055 / \mathrm{s}-0040-1718474$ ISSN 2277-954X.
(C2020. Neurotrauma Society of India.

This is an open access article published by Thieme under the terms of the Creative Commons Attribution-NonDerivative-NonCommercial-License, permitting copying and reproduction so long as the original work is given appropriate credit. Contents may not be used for commercial purposes, or adapted, remixed, transformed or built upon. (https://creativecommons.org/licenses/by-nc-nd/4.0/).

Thieme Medical and Scientific Publishers Pvt. Ltd. A-12, 2nd Floor, Sector 2, Noida-201301 UP, India 
of D2 spinous process, and bilateral posterior basal subsegmental consolidative pulmonary changes, likely aspiration pneumonia (-Fig. 1). Initial blood investigations showed white blood cells $12.58 \times 10^{3} / \mathrm{mm}^{3}$ with neutrophils $41.8 \%$, while other parameters were normal.

As a part of COVID-19 pandemic protocol in our hospital, all emergency cases were screened for a potential infection where patients with acute infections were considered as positive with all related consequences until proven otherwise. The patient was isolated and a nasopharyngeal swab for SARS-CoV-2 reverse transcription-polymerase chain reaction (RT-PCR) was taken in a negative pressure room that confirmed the patient infection with the virus. The patient was admitted in COVID-19 area in the intensive care unit (ICU).

The patient was managed in ICU with head elevation of 30 degrees. Mannitol, prophylactic antibiotics, analgesics, and prophylactic anticoagulation were prescribed. CSF leak from the left ear stopped on the second day post admission. Strict infection control measures were taken during dealing with the patient. A proper and effective personal protective equipment (PPE) donning before entering to the patient was done in the following order: perform hand hygiene, put on gown, surgical mask, face shield, then gloves. Safe PPE doffing was done in the following order: remove gloves, remove gown, healthcare personnel may exit patient room at this step, perform hand hygiene, remove face shield, remove and discard face mask, then perform hand hygiene. Infection control measures also extended to involve all body fluids, secretions and drainage fluids, as it is considered a potential source for COVID-19 infection, so a special care was given during dealing with these materials ensuring safe waste disposal under strict contact and droplet precautions. A special care during aerosol
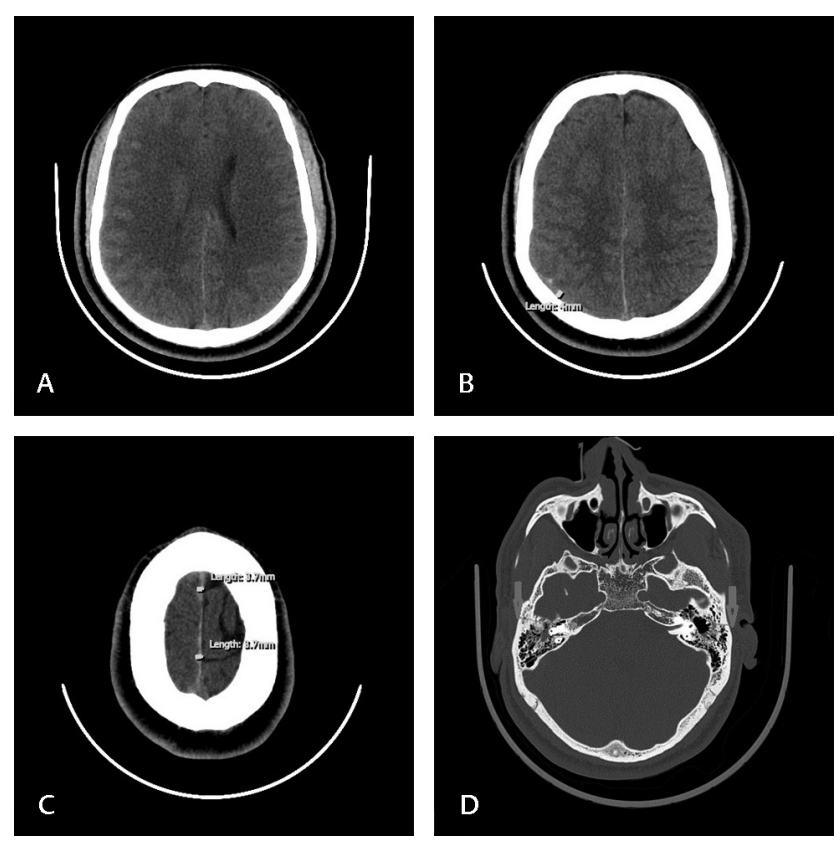

Fig. 1 Computed tomography brain axial cuts done on the presentation of the patient showing $(\mathbf{A})$ right-sided cerebral edema, (B) right temporoparietal subarachnoid hemorrhage, $(\mathbf{C})$ right temporal and parietal small brain contusions, and (D) bone window showing bilateral petrous bones longitudinal fractures (arrows). generating procedures was taken with effective N95 wearing ensuring proper ceiling technique.

The patient's conscious level improved gradually during the ICU stay. The facial nerve was intact bilaterally. CT brain follow-up on the fourth day of his admission revealed resolution of the subarachnoid hemorrhage, stable appearance of the cerebral contusions, regressive course of the cerebral brain edema, and newly depicted right tentorial leaflet thin subdural hematoma reaching the falx cerebri. Six days later, CT brain follow-up revealed improvement in the cerebral contusions, almost complete resolution of the cerebral brain edema, stable appearance of right tentorial leaflet thin subdural hematoma, and stable appearance regarding the bilateral petrous bone longitudinal fractures (-Fig. 2).

The patient was treated according to the hospital protocol for COVID-19. He regained his consciousness after 10 days and was sifted from ICU to ward at the 14th day post admission. At that time as per WHO guidelines, hospital policy, and after clinical evaluation, patient was cleared from isolation and announced as recovered COVID-19 case to continue taking the neurosurgical care in routine nonisolated bed.

Pure tone audiometry was done on the 16th day during hospital stay, after which he regained his consciousness. It showed severe sensorineural hearing loss (SNHL) on the right side and moderate SNHL on the left side. Hearing aid was advised if the hearing loss did not improve on follow-up.

The patient was discharged on the 18th day of hospital stay. High index of suspicion was the clue in this patient to start early treatment to achieve a good patient outcome and avoid viral spread to the staff and other admitted patients.
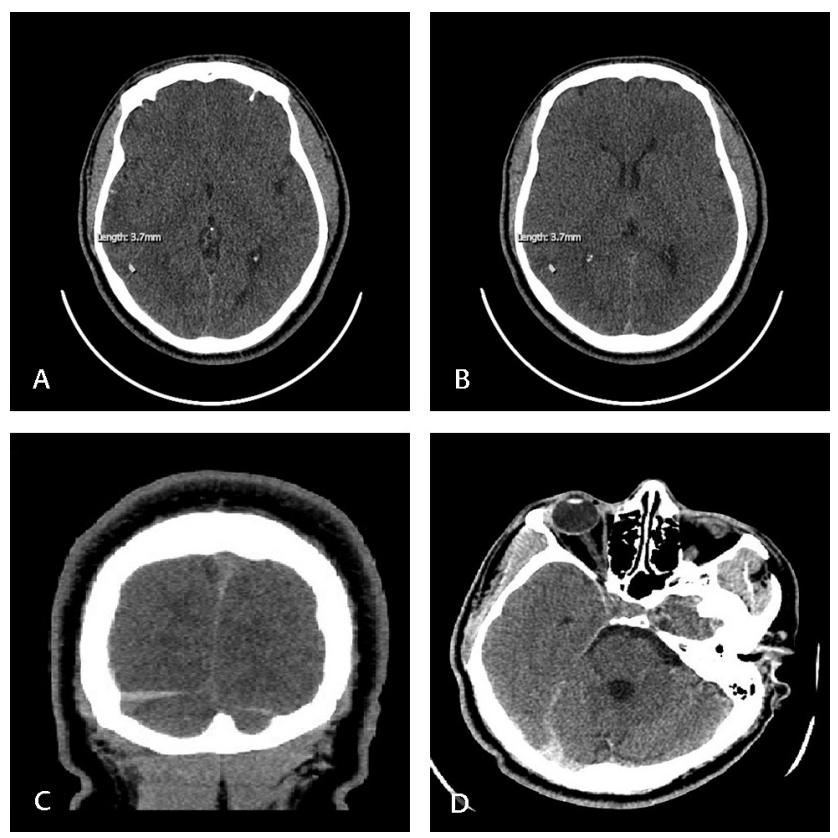

Fig. 2 Computed tomography $(C T)$ brain axial cuts done on the fourth day of the hospital stay showing $(\mathbf{A})$ and $(\mathbf{B})$ resolution of the previously noted right-sided subarachnoid hemorrhage, improvement in the right-sided cerebral brain edema, and stable appearance of the right temporoparietal small cerebral contusions; (C) newly depicted right tentorial leaflet thin subdural hematoma reaching the falx cerebri; and (D) CT brain axial cuts done on the 10th day showing stable appearance of right tentorial leaflet thin subdural hematoma. 


\section{Discussion}

Temporal bone fractures represent 14 to $22 \%$ of all skull fractures. Males are three to four times more prone to trauma than females. ${ }^{14}$ Around $90 \%$ of these fractures in adults are associated with intracranial injuries and $9 \%$ with cervical spine injuries. These fractures are bilateral in 8 to $29 \%$ of cases. ${ }^{1,5,6}$ Common complications of temporal bone fractures include facial nerve palsy, CSF leak, SNHL, conductive hearing loss, cholesteatoma formation, tympanic membrane perforation, hemotympanum, injuries to lower cranial nerves, and vascular injuries.

Our patient had bilateral longitudinal petrous fractures. Traditionally, temporal bone fractures have been classified into longitudinal, transverse, and oblique (mixed) fractures. Subsequently, they have been classified into otic capsule sparing and otic capsule disrupting (OCD) fractures, as such classification correlates well with the risk of concurrent functional complications. OCD fractures are associated with higher incidence of SNHL (sevenfold in one study), CSF leak (2-4fold), and facial nerve injury (30-50\%). ${ }^{6}$ High-resolution CT of the temporal bones is indicated when there is acute onset facial nerve paralysis, suspicion of an acute vascular injury, or another reason to prepare the patient for immediate or potential neurosurgical intervention. ${ }^{2}$

The measures taken to stem the COVID-19 pandemic had influenced the neurosurgical workflow, which tries to maximize the capability to provide neurosurgical care to the patients while reducing the risk for health-care providers to a minimum. ${ }^{7}$ Special precautions should be considered when dealing with suspected or confirmed COVID-19 patient with strict adherence to the recommended infection control measures. These measures include patient isolation, proper PPE donning and doffing, and proper hand hygiene. High index of suspicion of COVID-19 infection is important while dealing with any patient with special concern to the surgical and traumatized patients to reach a good patient outcome especially during the pandemic of COVID-19. ${ }^{8}$

Our patient had moderate head injury (GCS 9-12). Traumatic brain injury (TBI) remains the most common cause of posttraumatic mortality worldwide with considerable economic and social impact. ${ }^{9}$ TBI usually requires prompt surgical care and delaying treatment may negatively affect the outcome. ${ }^{7}$ The patient in our report was managed successfully conservatively. The influence of COVID-19 on TBI patients is currently unknown. Notably, both conditions lead to a coagulopathy.$^{10}$ However, pneumonia during the acute setting leads most likely to a worse outcome and increased length of stay. ${ }^{11}$

Our patient was diagnosed as COVID-19 positive through nasopharyngeal swab. They are based on RT-PCR to identify SARS-CoV-2. The disadvantage of these tests is that there is a non-negligible rate of false negatives, ranging from 4 to $30 \%$, depending on the expertise and training of those performing the swabs..$^{12}$ The execution of two nasopharyngeal swabs, distanced by at least 2 to 4 days, is recommended to improve the sensitivity of the method. Moreover, the accuracy of the swab test can be increased if complemented with chest imaging showing signs of infection. It was suggested that chest CT could be even more sensitive in detecting COVID-19 than repeated RT-PCR test. ${ }^{13}$

We used prophylactic antibiotics in our patient to guard against the risk of meningitis due to CSF leak. CSF leak occurs in 15 to $20 \%$ of all temporal bone fractures, which is more frequent with OCD fractures. The management of CSF leak in these patients is mainly through conservative measures. A meta-analysis revealed a statistically significant reduction in meningitis using prophylactic antibiotics in patients with CSF leak. ${ }^{14}$ Spontaneous resolution with conservative management occurs in $~ 95$ to $100 \%$ of patients. Surgical repair is recommended for those cases with persistent CSF leak 7 to 10 days after the injury. ${ }^{1}$

Our patient had bilateral SNHL, which was severe on the right side and moderate on the left side. The mechanisms involved in the development of SNHL involve avulsion of the cochlear nerve, disruption of the membranous labyrinth, interruption of the cochlear blood supply, cochlear hemorrhage, perilymph fistula, and endolymphatic hydrops..$^{15}$ The prognosis for patients with posttraumatic brain injury SNHL is very poor. Bone-anchored hearing aid has been demonstrated to have good outcomes for unilateral profound SNHL, while the patients with bilateral profound SNHL after temporal bone fractures may benefit from cochlear implantation.

\section{Conclusion}

The application of strict precautions of infection control during the management of bilateral temporal fractures in COVID-19-positive patients can ensure good outcomes.

\author{
Funding \\ None. \\ Conflict of Interest \\ None declared.
}

\section{References}

1 Brodie HA, Thompson TC. Management of complications from 820 temporal bone fractures. Am J Otol 1997;18(2):188-197

2 Diaz RC, Cervenka B, Brodie HA. Treatment of temporal bone fractures. J Neurol Surg B Skull Base 2016;77(5):419-429

3 Kowalski LP, Sanabria A, Ridge JA, et al. COVID-19 pandemic: effects and evidence-based recommendations for otolaryngology and head and neck surgery practice. Head Neck 2020;42(6):1259-1267

4 Exadaktylos AK, Sclabas GM, Nuyens M, et al. The clinical correlation of temporal bone fractures and spiral computed tomographic scan: a prospective and consecutive study at a level I trauma center. J Trauma 2003;55(4):704-706

5 Griffin JE, Altenau MM, Schaefer SD. Bilateral longitudinal temporal bone fractures: a retrospective review of seventeen cases. Laryngoscope 1979;89(9 Pt 1):1432-1435

6 Natarajan K, Madhav K, Saraswathi AV, Kameswaran M. Bilateral temporal bone fractures: a case report. Int J Otorhinolaryngol Head Neck Surg 2018;4(1):271-275

7 Eichberg DG, Shah AH, Luther EM, et al. Letter: academic neurosurgery department response to COVID-19 pandemic: the university of Miami/ Jackson memorial hospital model. Neurosurgery 2020;87(1):E63-E65 
8 Al-Jabir A, Kerwan A, Nicola M, et al. Impact of the coronavirus (COVID-19) pandemic on surgical practice - Part 1. Int J Surg 2020;79:168-179

9 Rubiano AM, Carney N, Chesnut R, Puyana JC. Global neurotrauma research challenges and opportunities. Nature 2015;527(7578):S193-S197

10 Samuels JM, Moore EE, Silliman CC, et al. Severe traumatic brain injury is associated with a unique coagulopathy phenotype. J Trauma Acute Care Surg 2019;86(4):686-693

11 Kumar RG, Kesinger MR, Juengst SB, et al. Effects of hospital-acquired pneumonia on long-term recovery and hospital resource utilization following moderate to severe traumatic brain injury. J Trauma Acute Care Surg 2020;88(4):491-500
12 Patel ZM. Reflections and new developments within the COVID-19 pandemic. Int Forum Allergy Rhinol 2020; 10(5):587-588

13 Ai T, Yang Z, Hou H, et al. Correlation of chest CT and RT-PCR testing for coronavirus disease 2019 (COVID-19) in China: a report of 1014 cases. Radiology 2020;296(2):E32-E40

14 Brodie HA. Prophylactic antibiotics for posttraumatic cerebrospinal fluid fistulae. A meta-analysis. Arch Otolaryngol Head Neck Surg 1997;123(7):749-752

15 Rizvi SS, Gibbin KP. Effect of transverse temporal bone fracture on the fluid compartment of the inner ear. Ann Otol Rhinol Laryngol 1979;88(Pt 1):741-748 